\title{
THE PERIPHERAL VASCULAR SYSTEM IN THE BULBAR CONJUNCTIVA OF YOUNG NORMOTENSIVE ADULTS AT REST ${ }^{1}$
}

\author{
By RICHARD E. LEE 2 and ELIZABETH A. HOLZE 2
}

(From the Department of Pharmacology, College of Medicine, Syracuse University, Syracuse, $N . Y$.)

(Submitted for publication June 24, 1949)

The recent development of specialized direct techniques for observation and measurement has added greatly to knowledge concerning the anatomy and function of the capillary bed in several species of small mammals (1-5). The spatial arrangement of vascular elements, vessel sizes, and relative velocities of blood flow have been established. In addition, the nature of spontaneous vasomotor activity, the relative sensitivity to vasoconstrictor agents, and other criteria, have been found suitable for the determination and comparison of peripheral vascular function in certain different experimental conditions (6-8). There has been little direct information obtained, however, regarding the reactivity and patterns of response to stimuli in the capillary blood vessels of man. This has resulted primarily from a lack of suitable techniques and the inaccessability of readily visualized complete peripheral vascular networks in the unanesthetized human. The present study is an attempt to apply some of the methods found useful in the experimental animal to observation and evaluation of the human capillary bed. With suitable apparatus, it was noted that the terminal vessels in the bulbar conjunctiva could be observed without difficulty and photographed at relatively high magnification. In addition, their exposed position allowed measurement of peripheral vascular reactions to a vasoconstrictor agent (epinephrine) applied directly to the area studied in known concentration. This procedure, based upon a knowledge of the anatomy in the capillary bed, permitted a comparison of the relative sensitivity of arterioles, precapillaries, and venules to a vasoconstrictor stimulus.

1 This study was supported by grants from the American Philosophical Society, Philadelphia, Pa., and the Nutrition Foundation, New York, N. Y.

2 Present address: The Department of Medicine, Cornell University Medical College, and The New York Hospital, New York, N. Y.

\section{METHODS}

A standard type of slit-lamp microscope allowed visualization of the bulbar conjunctiva up to magnifications of $62 \times$, with the subject sitting erect. Following reassurance, the head was placed in a support with the eyes fixed on a preselected site to afford study of the desired area and prevent undue movement. The bulbar conjunctiva of each eye was then examined under low power, and representative fields selected for further observation at higher magnification. The topography of vascular elements was studied and occasional areas photographed for further review. Speed of blood flow was compared in different vessels, and observations were made on the occurrence and rates of spontaneous vasomotion (variations in caliber of arterioles, and opening and closing of precapillary sphincters). Finally, threshold concentrations of epinephrine were determined which when applied directly to the region would just produce contraction to the point of closure of the precapillary sphincters. During these determinations, the epinephrine in Ringer's solution was maintained at a temperature of 36-37 degrees Centigrade.

The studies were carried out on 51 young adults from 21 to 34 years old. Thirty-three were male, and 18 were female. None were hypertensive, although one subject had had a markedly "positive" response to a standardized cold pressor test about six months previously. There was no noted abnormality in the arteriolar responses in this individual.

\section{RESULTS}

\section{General topography}

The arrangement of terminal arterioles, capillaries and venules in the human conjunctiva was generally in accord with that previously described in the mesentery and omentum of the rat, dog, and guinea pig $(3,4,5,8)$ (Figure 1). Capillaries arose at intervals from the end arterioles and formed an irregular network of vessels which became confluent to form the venular system. The arterioles frequently terminated in main $\mathrm{A}-\mathrm{V}$ channels which communicated directly with the venular tree. This latter pattern was most notable near the corneo-scleral junction. 

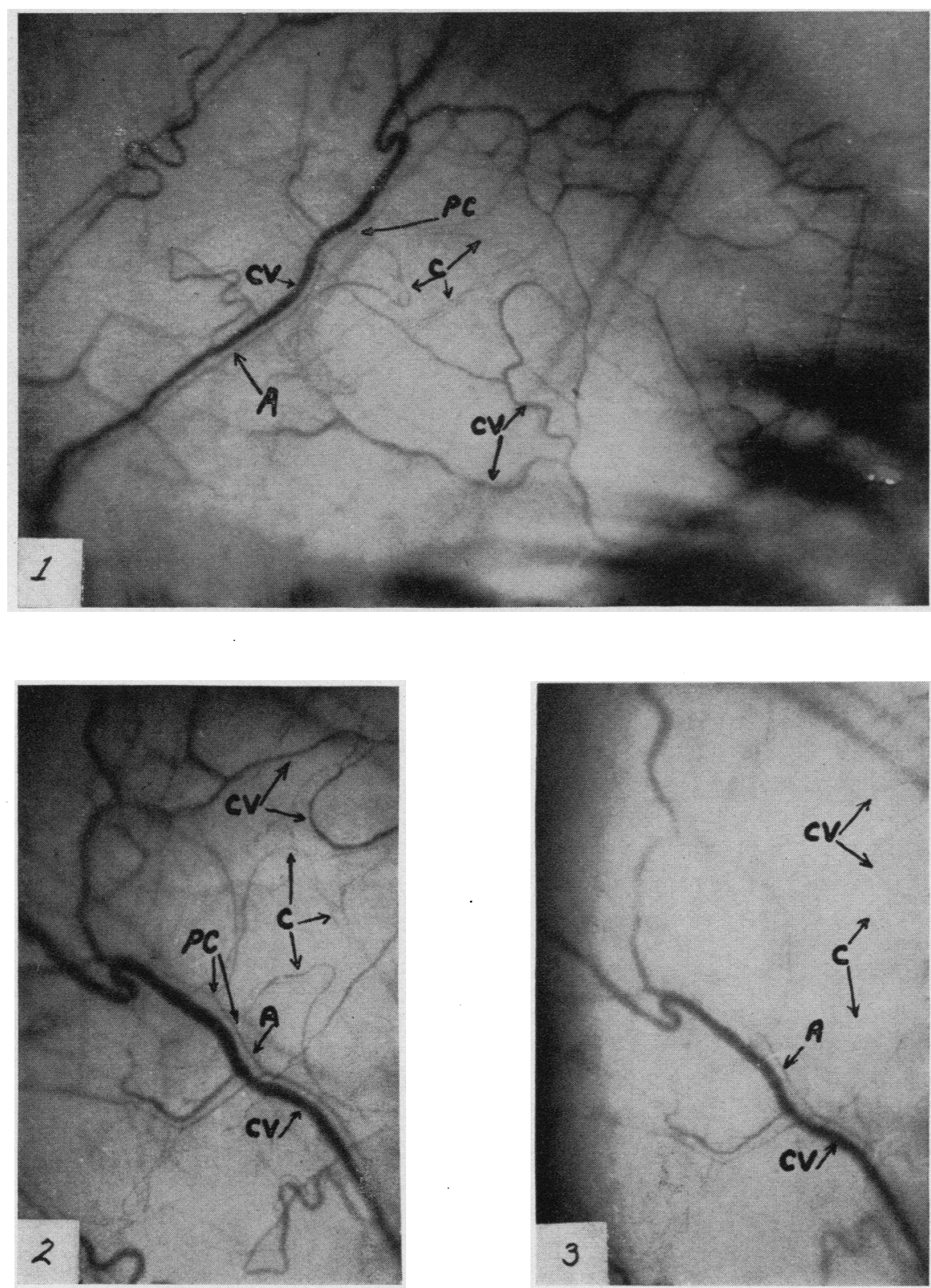

FIGs. 1-3.

Fig. 1. Conjunctiva $\times 60$, Showing the General Arrangement of Vessels in the Capillary Bed.

$\mathrm{CV}=$ collecting venules; $\mathrm{A}=$ arterioles; $\mathrm{C}=$ true capillaries, $\mathrm{PC}=$ precapillary region.

Fig. 2. Same Area (See Figure 1) $\times 80$, Indicating the Patent Capillary System, Most Notable on the Right Half of the Figure, Prior to the ApplicaTION OF EPINEPhrine to THE Conjunctiva.

Fig. 3. Same Region (See Figure 1 ) $\times 80$, Subsequent to the Administration of Epinephrine Solution, $1 / 30,000$, Locally.

Note the narrowing of arterioles and small venules; and the disappearance of many capillaries, due to the complete precapillary closure and drainage of residual capillary blood into the patent venular system. This degree of reaction is moderately greater than that often seen. 


\section{Spontaneous vasomotor activity}

At the magnification used it was often difficult to determine minor changes in diameter or flow in the arterioles. For, this reason, attention was directed primarily to the capillaries where relatively greater fluctuations in these phenomena could be readily seen. Here vasomotion was observed in approximately $20 \%$ of the vessels. Constriction invariably occurred at the most proximal portion where the capillary originated from the parent arteriole. In animals, this "precapillary" region has been shown to be the only portion of the true capillaries to possess smooth muscle cells $(3,4)$. At complete constriction, with interruption of capillary flow, the blood slowly drained into the collecting venules. Such a capillary would frequently remain closed for two to three minutes, then gradually open, with a return of blood flow lasting one to five minutes before the next contraction. These constricted and partially dilated phases of vasomotion were extremely irregular in degree and in duration; but in general the opened state tended to predominate.

\section{Speed of blood flow, in the various vessels}

In 10 subjects, attempts were made with a stopwatch and ocular micrometer to measure the speed of blood flow within the arterioles, capillaries and venules. Because of the usual tortuosity, determinations of length were not possible in each vessel. Only those were used whose course was approximately straight over a distance of 0.05 to 0.3 $\mathrm{mm}$. where velocities of single red cells could be timed.

Arterioles: The opaque wall and relatively rapid flow within the arterioles slightly obscured the corpuscular stream and possibly interfered to a moderate degree with the accuracy of absolute values obtained for flow in many of these elements. However, ail observations made on flow within those terminal arterioles of 10-14 micra in diameter were between 0.09 and $0.16 \mathrm{~mm}$./sec. (Table I).

TABLE I

Velocity of blood flow in the bulbar conjunctival vessels (mm./sec.; 10 subjects)

Arterioles Capillaries

$0.09-0.16(0.12$ av. $)$ 0.009-0.04 (0.026 av.)

Venules

$0.046-0.07$ (0.056 av.)
Capillaries: Capillary blood flow, on the other hand, was easily visualized. It was considerably slower than that in the arterioles, with a mean rate of $0.026 \mathrm{~mm}$./ $/ \mathrm{sec}$. The spontaneous changes in caliber of the precapillary portion resulted in variations of capillary flow speed from 0.009 to $0.040 \mathrm{~mm}$./sec. Capillary length averaged 0.065 $\mathrm{mm}$. Therefore, in the conjunctiva, the red blood cell spent approximately 2.5 seconds in traversing the capillary bed per se. During hyperemia and congestion (induced by light massage of the eyeball) this speed was often reduced to about onethird that existing previously.

Venules: After entering the system of collecting venules, the speed of blood flow began to increase, with an average rate of $0.056 \mathrm{~mm}$./ sec. found in venules of $12-20$ micra in diameter. Venular flow, in contrast to that within the capillaries, was consistently more regular as a result of the continued supply of blood during capillary closure by the main A-V channels. The patency of these elements during widespread capillary ischemia allowed relatively large volumes of blood to by-pass the capillary bed and enter the venular system directly.

\section{Responses to topically applied epinephrine}

Beginning with weaker solutions and progressing to the more concentrated, four drops of epinephrine in Ringer's solution were placed beneath the lower lid, and the eye closed for 15 seconds. Vessels previously selected were examined for vasoconstriction after each application of the agent. The first responses found in all cases were contraction of the precapillary region with slowing and occasionally stoppage of capillary blood flow, and a slight narrowing of the terminal arterioles in some instances. This endpoint was readily seen (Figures 2 and 3 ).

There was a close agreement found in the threshold reaction values of the subjects examined (Table II). All were included within the range of epinephrine concentrations $1 / 50,000$ to $1 / 20$,-

TABLE II

Average reaction threshold concentrations of epinephrine for bulbar conjunctival vessels (51 subjects)

Arterioles- 1:20,000

Precapillaries - $1: 35,000$ 
000 (using test dilutions of $1 / 100,000,1 / 50,000$, $1 / 35,000,1 / 20,000,1 / 15,000$, and $1 / 10,000)$.

The anatomical classes of peripheral vessels corresponded well with a differential reaction threshold of each to epinephrine. As in lower mammals, the precapillary sphincters were the most sensitive elements, constricting completely at stimuli that induced only a slight narrowing of the terminal arterioles and $\mathrm{A}-\mathrm{V}$ thoroughfares (Table II). The collecting venules, on the other hand, were the least reactive. They would usually respond at concentrations of epinephrine that also induced arteriolar narrowing. Such venular changes were slight, however, and were usually band-like and irregular in nature, in contrast to the more uniform manner of arteriolar constriction.

\section{DISCCSSION}

The topography of the peripheral vascular system in the human bulbar conjunctiva agrees closely with that previously described in the mesentery and omentum of certain small mammals $(3,5)$. This similarity in widely separated areas and in different species suggests that there is a relationship between the architecture of the vessel bed and the functions of its components common to all tissues. For example, the arrangement of capillary off shoots from the arteriolar tree allows complete closure of capillaries without greatly reducing venular return from the region. During widespread arteriolar and precapillary constriction with resulting cessation of capillary blood flow, in either the rat, dog, or man, blood still crosses from the arterioles to the venular bed through the patent $\mathrm{A}-\mathrm{V}$ channels. The capillary bed is cut off from the arteriolar blood supply; but venular flow, although reduced, is still maintained. Residual capillary blood is effectively drained through the patent venous end of the capillaries into the active venular circulation. Associated with this anatomical pattern in the capillary bed of the human conjunctiva, there is also a difference between the reactivity of smooth muscle cells forming the precapillary sphincters and those surrounding the arterioles. Stimuli which result in complete capillary occlusion and cessation of flow, may produce only a slight narrowing of the parent arteriole, without complete interruption of flow to the venules. Therefore, should tissue masses be subject to widespread capillary ischemia, one can assume that this anatomical arrangement of vessels and their differential responsiveness to stimuli may permit the return to the central vascular system and redistribution to other tissues of large volumes of otherwise stagnant capillary blood.

In addition to their high reactivity to epinephrine, the precapillary portions were also the most active regions with regard to spontaneous vasomotion. This is in accord with previous findings in other species $(3,5)$, although in man the phenomenon was seen less frequently, and in active vessels it was relatively slower in rate. Accurate comparisons are difficult, however, because of the irregularities in duration of each phase (constricted $v$ s. dilated). The significance of spontaneous vasomotor activity in the hemodynamics of capillary blood flow has been fully discussed elsewhere (9).

Although the nature of either spontaneous or induced vascular responses in the conjunctival vessels of man follows closely that observed in the mesenteric areas of other forms, the absolute threshold levels of reaction to epinephrine in humans are notably lower. The precapillary sphincters of the rat meso-appendix and the dog omentum usually constrict or completely close in response to epinephrine solutions of $1 / 2,000,000$ (3). This contrasts sharply with the absence of any observed precapillary changes in the conjunctiva of subjects in this study at concentrations weaker than 1/50,000. Data on the responsiveness of bulbar conjunctival vessels of other species are not available. The difference found could conceivably result from dissimilar techniques of applying the solution, the erect position used in examining humans as opposed to the horizontal plane assumed by the mesentery and omentum, or possible permeability differences between the exposed bulbar membranes and the mesenteric regions observed in other investigations.

\section{SUMMARY AND CONCLUSIONS}

1. The bulbar conjunctival capillary bed in man can be readily visualized with the microscope at relatively high magnification. The arrangement of vessel elements, the velocity of blood flow, and the nature of spontaneous vasomotor activity in the conjunctival vessels have been observed and described. These features of the peripheral vascu- 
lar system in humans are in accord with those observed in lower mammals.

2. The threshold reactivity to topically applied epinephrine in the terminal arterioles and precapillary sphincters of this region was found to be constant under the conditions described.

3. As with other mammals, the precapillary sphincters were the most sensitive vessels to epinephrine in the capillary bed, with the small arterioles and the collecting venules relatively less so in that order.

4. The possible significance of vascular topography and pattern of response to stimulation is briefly discussed with regard to the hemodynamics of the peripheral circulation as seen in the bulbar conjunctiva.

5. The epinephrine test, as described, offers a means of determining directly the sensitivity of conjunctival arterioles in man to a quantitative vasoconstrictor stimulus.

\section{BIBLIOGRAPHY}

1. Clark, E. R., and Clark, E. L., Caliber changes in minute blood-vessels observed in the living mammal. Am. J. Anat., 1943, 73, 215.
2. Knisely, M., The histophysiology of peripheral vascular beds, in: Blood, Heart, and Circulation. Am. Assoc. Adv. of Sci. Publ. 303, Science Press, Lancaster, Pa., 1940, p. 303.

3. Chambers, R., and Zweifach, B. W., Topography and function of the mesenteric capillary circulation. Am. J. Anat., 1944, 75, 173.

4. Zweifach, B. W., A micro-manipulative study of blood capillaries. Anat. Rec., 1934, 59, 83.

5. Lee, Richard E., and Lee, Nina Z., The peripheral vascular system and its reactions in scurvy: an experimental study. Am. J. Physiol., 1947, 149, 465.

6. Zweifach, B., and Shorr, E., Hepato-renal factors in circulatory homeostasis. XXVI. Effect of adrenalectomy on renal VEM system and hypertension. Federation Proc., 1949, 8, 175.

7. Crismon, J. M., and Fuhrman, F. A., Studies on gangrene following cold injury. VI. Capillary blood flow after cold injury, effects of rapid warming, and sympathetic block. J. Clin. Invest., 1947, 26, 468.

8. Zweifach, B. W., Lee, R. E., Hyman, C., and Chambers, R., The omental circulation in morphinized dogs subjected to graded hemorrhage. Ann. Surg., 1944, 120, 232.

9. Chambers, R., Vasomotion in the hemodynamics of the blood capillary circulation. Ann. New York Acad. Sc., 1948, 49, 549. 